\title{
Anticancer efficacy of cisplatin and trichostatin A or 5-aza-2'-deoxycytidine on ovarian cancer
}

\author{
F Meng ${ }^{1,2}, \mathrm{G}$ Sun ${ }^{1,2}, \mathrm{M}$ Zhong $^{2}, \mathrm{Y} Y \mathrm{Yu}^{*, 2}$ and M A Brewer ${ }^{*, 1}$ \\ ${ }^{1}$ Division of Gynecologic Oncology, University of Connecticut Health Center, 263 Farmington Avenue, Farmington, CT 06030, \\ USA and ${ }^{2}$ Department of Obstetrics and Gynecology, Nanfang Hospital, Southern Medical University, 1838 North Guangzhou \\ Avenue, Guangzhou 510515, China
}

Background: To evaluate the anticancer efficacy of the combination of epigenetic modifiers and cisplatin in human ovarian cancer.

Methods: The effect of trichostatin A (TSA) and 5-aza-2'-deoxycytidine alone or in combination with low-dose cisplatin was evaluated on human ovarian cancer cell lines in vitro. We measured drug interaction by MTS assay, migration by transwell assay, expression of epithelial to mesenchymal transition (EMT) markers (Twist, Snail, Slug, E-cadherin, and N-cadherin), pluripotency markers (Oct4, Sox2, and Nanog), and epigenetic markers (DNMT3A, LSD1 and H3K4me2, H3K4me3, H3K9me2, and H3K9me3) by western blot, and the impact on and characteristics of spheroid growth when exposed to these drugs. Mouse xenografts were used to evaluate the anticancer effect of sequential drug treatment.

Results: Combination treatment had greater efficacy than single drugs and significantly suppressed cell viability, migration, and spheroid formation and growth. Sequential treatment of cisplatin $\left(1 \mathrm{mg} \mathrm{kg}^{-1}\right)$ followed by TSA $\left(0.3 \mathrm{mg} \mathrm{kg}^{-1}\right)$ significantly suppressed tumorigenicity of HEY xenografts through inhibition of EMT and decreased pluripotency of ovarian cancer cells.

Conclusion: Epigenetic modifiers potentiate the anticancer efficacy of low-dose cisplatin in ovarian cancer through regulation of EMT and pluripotency, and may provide a promising treatment for ovarian cancer patients.

Ovarian cancer is the most lethal malignancy of the female reproductive system, resulting in 22280 new cases and 15500 deaths in the United States in 2011 (Siegel et al, 2012). The most common therapy is cytoreductive surgery followed by platinumbased chemotherapy resulting in a response in more than $80 \%$ of patients (Vaughan et al, 2011). However, many patients will relapse within 5 years and ultimately die of chemoresistance (Yap et al, 2009). The ability to improve chemosensitivity would result in significant improvement in the treatment of and outcome of ovarian cancer. Ovarian cancer remains one of the most difficult cancers to detect early, possibly because aggressive ovarian cancers metastasise before emergence of an ovarian lesion that can be easily identified (Bast et al, 2009).
Ovarian cancer has a unique multi-organ metastatic pattern, in which localised cancer cells detach, acquire motility, metastasise as spheroids, escape immunological surveillance, attach to distant organ surfaces, and invade to develop tumour implants (Lengyel, 2010). Ovarian cancer spheroids are thought to have an important role in the dissemination of ovarian cancer, with the ability to invade into mesothelial cells that line the peritoneal cavity. The cells within a spheroid acquire more aggressive features than are present in primary tumour cells (Ahmed et al, 2007). Ovarian cancer cells that have the capability of forming spheroids show contractile behaviour mediated by actomyosin, which compacts the spheroid, promoting cell migration (Sodek et al, 2009) and may have a role in chemoresistance. Prevention of spheroid formation 
would present a new and promising strategy for promoting chemosensitivity.

Epigenetic aberrations, including DNA methylation, histone modifications, and microRNA dysregulation are now well established in the development and progression of ovarian cancer, and their gradual accumulation is associated with advancing disease stage and grade (Balch et al, 2009; Asadollahi et al, 2010). Epigenetic aberrations are relatively stable and are present in circulating serum, representing promising diagnostic, prognostic, and pharmacodynamic biomarkers ( $\mathrm{Su}$ et al, 2009; Teschendorff et al, 2009). In contrast to DNA mutations and deletions, aberrant gene-repressive epigenetic modifications are potentially reversible by epigenetic modulating therapies, including inhibitors of DNA methylation or histone-modifying enzymes (Kristensen et al, 2009). Although epigenetic monotherapies have not shown significant activity against most solid tumours (Kelly et al, 2010), preclinical studies suggest that they will be effective when used in combination with one another or with conventional chemotherapeutics, and combinatorial epigenetic therapy regiments are being examined in clinical trials (Plimack et al, 2007; Kristensen et al, 2009).

In this study, we found that trichostatin A (TSA), a histone deacetylase inhibitor, 5-aza-2'-deoxycitidine, a DNA methyltransferase inhibitor, and cisplatin, a DNA crosslinking agent, commonly used to treat ovarian cancer, alone or in combination significantly suppressed spheroid formation and growth of ovarian cancer cells in vitro, and sequential treatment of epigenetic modifiers and low-dose cisplatin reduced tumorigenesis more effectively than either drug alone in xenograft mouse models. These observations provide us a new prospect for treatment of resistant ovarian cancer.

\section{MATERIALS AND METHODS}

Cell lines and chemotherapeutics. Human ovarian cancer cell lines HEY, SKOV3, and the normal cell line IOSE were purchased from American Type Culture Collection (ATCC, Manassas, VA, USA). The A2780 cell line was a generous gift from Dr Susan Murphy (Obstetrics and Gynaecology, Duke University Medical Center, NC, USA). Cell lines were cultured in DMEM/F-12 medium (Invitrogen, Grand Island, NY, USA) containing 10\% fetal bovine serum (Cellgro) and 1\% Penicillin G/Streptomycin (Invitrogen). Chemotherapeutics used were TSA $\left(5 \mathrm{~mm} / 200 \mu \mathrm{l}^{-1}\right)$, 5-aza-2'-deoxycytidine (10 mg, 5-aza-CdR) (Sigma-Aldrich, St Louis, MO, USA), and cisplatin (100 mg $100 \mathrm{ml}^{-1}$, Ben Venu Laboratories, Bedford, OH, USA).

Cytotoxicity assay. Cell viability was assessed using Promega Cell Proliferation MTS Assay (Promega, Madison, WI, USA). Briefly, ovarian cancer cells were seeded in 96-well plates at a density of $1 \times 10^{3}$ per well and incubated with increasing concentrations of TSA, 5-aza-CdR, or cisplatin alone for $48 \mathrm{~h}$ to determine a doseresponse curve. Doses were fixed based on the time course (TSA $300 \mathrm{~nm}, 5$-aza-CdR $10 \mu \mathrm{M}$, and cisplatin $1 \mu \mathrm{M}$ ), and cytotoxicity at different time points $(24-96 \mathrm{~h})$ was measured to determine the optimal incubation time. In order to investigate if the epigenetic modifiers could enhance the cytotoxicity of cisplatin, we determined cell viability of HEY cell in the presence of a fixed dose of cisplatin $(1 \mu \mathrm{M})$ in combination with increasing doses of TSA (1-600 nM) or 5-aza-CdR (from 1 to $50 \mu \mathrm{M}$ ) for $48 \mathrm{~h}$ in vitro. Subsequently, dead cells were washed away, the attached cells were incubated with MTS, and cell viability was detected using Microreader Model-680 (Bio-Rad, Hercules, CA, USA). All the experiments were done in triplicate.

Drug interaction measurement. Promega Cell Proliferation MTS Assay was used for assessment of synergism between drugs. The
Chou-Talalay median effect and combination index (CI) model was used to determine if there was synergism between epigenetic modifiers and cisplatin (Chou, 2010). Briefly, SKOV3 cells were treated with each drug individually at multiples (1.0, 2.0, and 3.0) and a fraction $(0.5)$ of the $\mathrm{IC}_{50}$ concentration in a fixed ratio (TSA/ cisplatin $=0.3 / 1 ; 5$-aza-CdR/cisplatin $=10 / 1)$ for $48 \mathrm{~h}$ in vitro. Combination index was calculated as: $C I=(D)_{1} /\left(D_{X}\right)_{1}+(D)_{2} /$ $\left(D_{X}\right)_{2} \cdot\left(D_{X}\right)_{1}$ and $\left(D_{X}\right)_{2}$ are the concentration of single drugs required to inhibit $x \%$ of cells, and $(D)_{1}$ and $(D)_{2}$ are drug concentrations of the combination treatment that inhibits $\mathrm{x} \%$ of cells. Combination index $<1, \mathrm{CI}=1$, and $\mathrm{CI}>1$ indicate drug synergism, additive effect, and antagonism, respectively.

Cell migration assay. In vitro cellular migration was measured by determining the ability of cells to migrate through a transwell membrane using the Transwell permeable support system (Corning, Coring, NY, USA). Three cancer cell lines were treated with TSA, 5-aza-CdR, and cisplatin alone or in combination for $48 \mathrm{~h}$ in vitro, then digested and seeded in the transwell chamber for $48 \mathrm{~h}$. Transwell membranes were then washed with PBS, fixed with formaldehyde, stained with H\&E stain, and five fields of each group were selected for analysis. The number of cells capable of migrating through the permeable membrane were counted and the untreated control was compared with each treatment group.

Western blotting. Monolayer cells, cells derived from tumour xenografts or spheroids, were lysed and total protein was quantitated using Bradford Protein Assay (Bio-Rad). Protein was denatured in $2 \times$ SDS-PAGE sample buffer, $10-30 \mu \mathrm{g}$ per lane samples were loaded and separated on $7-10 \%$ SDS-PAGE gel, the gels were transferred to nitrocellulose membrane, membranes were blocked in $5 \%$ non-fat milk for $1 \mathrm{~h}$, and incubated with primary antibodies at $4{ }^{\circ} \mathrm{C}$ overnight. The antibodies and dilution were: E-cadherin, N-cadherin, Twist, Slug, Snail, Oct4, Nanog, Sox2, DNMT3A, LSD1 $(1: 1000$, Cell Signalling Technology, Danvers, MA, USA), and $\beta$-actin as loading control (1:1000, SigmaAldrich). The membranes were washed with TBST three times for $10 \mathrm{~min}$, incubated with secondary antibodies $(1: 1000$, Goat anti mouse IgG-HRP or Goat anti rabbit IgG-HRP or Bovine anti goat IgG-HRP, Santa Cruz Biotechnology, Santa Cruz, CA, USA) for $1 \mathrm{~h}$ at room temperature, and washed with TBST three times for $10 \mathrm{~min}$. Chemiluminescent substrate was added to the membranes and the expression of proteins was detected by G:box \& Genesys system (Syngene, Frederick, MD, USA).

Histone immunoblots. Histone protein was extracted and purified by Epiquick total histone extraction kit (Epigentek, Farmingdale, NY, USA). The extraction was quantified, diluted in $1 \times$ NuPAGE/LDS loading buffer (Invitrogen), heated in $95^{\circ} \mathrm{C}$ dry heater (Labnet, Woodbridge, NJ, USA) for $5 \mathrm{~min}$, and loaded in $10 \% \mathrm{NuPAGE} / \mathrm{Bis} / \mathrm{Tris}$ gel (Invitrogen) with NuPAGE/MES/SDS buffer (Invitrogen). The protein was transferred onto nitrocellulose membranes, blocked in 5\% BSA/TBST solution for $1 \mathrm{~h}$, and incubated with primary antibody at $4{ }^{\circ} \mathrm{C}$ overnight. After washing with $1 \times$ TBST three times for $10 \mathrm{~min}$, secondary antibodies were added and the subsequent protocol was identical to the western blotting (paragraph above). The antibodies and dilution were: H3K4me2, H3K4me3, H3K9me2,H3K9me3, and Histone H3 ( $1: 1000$, Cell Signalling Technology).

Spheroid formation and growth assay. Human samples were collected from patients at the time of surgery after written informed consent, and the protocol was approved by the Institutional Review Board of The University of Connecticut Health Center. Spheroids were isolated from fresh human ovarian cancer ascites and photographed with an IX71 Olympus microscope (Olympus, Center Valley, PA, USA). Suspended single cells from cell lines (500 cells per $\mathrm{ml}$ ) were seeded in 24-well Corning Ultra-Low Attachment Plates (Corning) in MammoCult Basic 
Medium and MammoCult proliferation supplements (Stemcell Technology, Vancouver, BC, Canada) and incubated at $37^{\circ} \mathrm{C}$ for 15 days. Two different protocols were followed in this assay. Spheroid formation assay was done by mixing suspended cells with TSA, 5-aza-CdR, and cisplatin alone or in combination whereas spheroid growth assay was done by adding the same dose of drugs into the spheroid culture medium after spheroids had been grown for 5 days. Briefly, spheroids were centrifuged at 1000 r.p.m., the supernatant was discarded, spheroids were then digested with $0.25 \%$ EDTA-trypsin for $10 \mathrm{~min}$, and the digested cells were resuspended in DMEM/F-12 medium. Cell numbers were counted by hemocytometer and cell viability was detected by Trypan blue dye (Sigma-Aldrich), using an IX71 Olympus microscope. To further investigate the spheroid formation ability (spheroid number), 1000 suspended cells were seeded with $\operatorname{drug}(\mathrm{s})$ in 96-well Corning Ultra-Low Attachment Plates and then incubated at $37^{\circ} \mathrm{C}$ for 15 days. The spheroid numbers were counted and compared between control and treatment groups using one-way ANOVA.

In vivo tumorigenicity assay. C3H.C-Prkdc/SCID mice (4-5 weeks old, female, weight: $18 \pm 0.5 \mathrm{~g}$ ) were used and the protocol were approved by the Institutional Animal Care and Use Committee of The University of Connecticut Health Center. Trichostatin A, 5-aza$\mathrm{CdR}$, and cisplatin alone or in combination were incubated with HEY cells $\left(10 \times 10^{6}\right)$ for $48 \mathrm{~h}$, and then injected into the mice subcutaneously. The tumour size was measured by caliper every 2 days and the mice were killed at day 22 , tumours were harvested and weighed.

In vivo anticancer assay. HEY cells $\left(5 \times 10^{6}\right)$ were injected subcutaneously into the mouse and treatment started when tumours reached $0.5 \mathrm{~cm} \times 0.5 \mathrm{~cm}$. Treatments were initiated with IP drug injection daily for 5 days, followed by a 2-day drug holiday. The second drug was then injected IP daily for 5 days. The drug dose of TSA, 5-aza-CdR, and cisplatin were $0.3,10$, and $1 \mathrm{mg} \mathrm{kg}^{-1}$, respectively, calculated with allometric scaling and mice were killed at day 26. Doses of TSA and 5-aza-CdR came from Phase I trials (Fang et al, 2010) and cisplatin was calculated to be equivalent to half the dose commonly administered to women (60-75 $\left.\mathrm{mg} \mathrm{M}^{-2}\right)$. Both tumour weights and the largest tumour diameter were measured, and tumour volume was calculated as described previously (Baba et al, 2009).

Statistical analysis. SPSS 16.0 software (IMB, Armonk, NY, USA) was used to analyse the data and GraphPad Prism 5 (GraphPad Software, San Diego, CA, USA) was used to create the graphs. The differences between groups were evaluated using one-way ANOVA, with $P<0.05$ being considered significant.

\section{RESULTS}

Effect of TSA, 5-aza-CdR, and cisplatin on ovarian cancer cell viability. Cell viability of SKOV3 cells, an ovarian cancer cell line, was assessed in the presence of TSA, 5-aza-CdR, or cisplatin (Figure 1A). A dose response for the three drugs was determined with doses ranging from $1 \mathrm{~nm}$ to $100 \mu \mathrm{M}$, and the $\mathrm{IC}_{50}$ of TSA, 5 -aza-CdR, and cisplatin was found to be $0.1,5$, and $1 \mu \mathrm{M}$, respectively (Figure 1B). Trichostatin A had the most cytoxicity and 5-aza-CdR had the least cytotoxicity but all drugs at high doses resulted in $100 \%$ non-viable cells. To further investigate if cell viability was affected by the exposure time of different drugs, a time course assay (24-96h) was done. Forty-eight hours was determined to be the optimal time of drug exposure because there was the maximal reduction in cell number at this time point for all three drugs (Supplementary Figure S1). Co-treatment with both TSA and cisplatin or 5-aza-CdR and cisplatin at a fixed dose $(0.3: 1$ and $10: 1$, respectively) in SKOV3 cells showed that the combination of $0.3 \mu \mathrm{M} \mathrm{TSA} / 1 \mu \mathrm{M}$ cisplatin $(\mathrm{CI}=0.424)$ and $10 \mu \mathrm{M}$ 5 -aza-CdR $/ 1 \mu \mathrm{M}$ of cisplatin $(\mathrm{CI}=0.454)$ achieved significant synergism and these two combinations were chosen for further studies (Figure 1C and D).

Combination of TSA and cisplatin inhibits cancer cell migration and enhances drug sensitivity than TSA, 5-aza-CdR, or cisplatin alone. The migration capability of ovarian cancer cells was measured after being treated with TSA, 5-aza-CdR, and cisplatin alone or in combination, and cell migration of all three cancer cell lines was significantly inhibited by TSA, 5-aza-CdR, or cisplatin alone as well as when cisplatin was combined with TSA or 5-aza-CdR (Figure 2A). The combination of TSA/cisplatin and 5-aza-CdR/cisplatin inhibited migration more than either drug alone. Trichostatin A/cisplatin was slightly superior to 5 -aza-CdR/cisplatin in all three cell lines HEY, SKOV3, and A2780 (Figure 2A). The highly chemoresistant cell line HEY had increasing sensitivity to low-dose cisplatin $(1 \mu \mathrm{M})$ with increasing doses of TSA and 5-aza-CdR (Figure 2B). To further confirm if the changes of migration and chemotherapy sensitivity were due to regulation of tumour cell pathways, multiple proteins that have a pivotal role in cell epithelial to mesenchymal transition (EMT) and pluripotency were measured. HEY cells, which have high expression of $\mathrm{N}$-cadherin and absent expression of E-cadherin, had the most downregulation of $\mathrm{N}$-cadherin with exposure to 5 -aza-CdR/cisplatin (Figure 2C). Inversely, E-cadherin is highly expressed in SKOV3 cells and was downregulated by exposure to both TSA/cisplatin and 5-aza-CdR/cisplatin (Figure 2C). $\mathrm{N}$-cadherin was expressed in SKOV3 cells and was also significantly downregulated with both combinations (Figure 2C). Nanog and Oct- 4 were significantly suppressed by the combination treatment but Sox 2 was minimally affected in SKOV3 cells (Figure 2C). The expression of Sox 2 was significantly suppressed by TSA/cisplatin in HEY cells (Figure 2C).

Epigenetic regulation of ovarian cancer cells in the presence of epigenetic modifiers and cisplatin. Epigenetic regulation and modification were evaluated when epigenetic modifiers were used in combination with cisplatin. DNMT3A, a suppressor of tumour suppressor genes, has been shown to be suppressed by DNMT inhibitors (Yoo, Jones 2006). In ascites-derived SKOV3 cells, TSA or 5-aza-CdR combined with cisplatin inhibited the expression of DNMT3A, compared with TSA or 5-aza-CdR alone (Figure 3A). Trichostatin A significantly suppressed the expression of DNMT3A, suggesting that there is a link between histone hyperacetylation and DNA methylation, as has been observed in previous studies (Cedar et al, 2009). Another epigenetic modification core enzyme LSD1, a specific histone demethylase, which demethylates $\mathrm{H} 3 \mathrm{~K} 4$ or $\mathrm{H} 3 \mathrm{~K}$, was significantly suppressed by both combination treatments in SKOV3 cells (Figure $3 \mathrm{~A}$ ). In order to investigate if histone methylation was affected, four methylation markers, related to gene transcription activity (H3K4me2 and $\mathrm{H} 3 \mathrm{~K} 4 \mathrm{me} 3$ ) and gene silencing (H3K9me2 and $\mathrm{H} 3 \mathrm{k} 9 \mathrm{me} 3$ ), were evaluated. Trichostatin A and 5-aza-CdR alone or in combination with cisplatin suppressed $\mathrm{H} 3 \mathrm{~K} 4 \mathrm{me} 2, \mathrm{H} 3 \mathrm{~K} 4 \mathrm{~m} 3$, and $\mathrm{H} 3 \mathrm{~K} 9 \mathrm{me} 3$ in SKOV3 cells, but had less effect on H3K9me2 (Figure 3B). A2780 cells, which are derived from a primary ovarian tumour, had different responses than SKOV3 cells. DNMT3A was moderately suppressed when treated with TSA/cisplatin and slightly suppressed with 5 -aza-CdR/cisplatin, with no significant change of LSD1 (Figure 3A). The methylation markers $\mathrm{H} 3 \mathrm{~K} 4 \mathrm{me} 3$ and H3K9me2 were suppressed by single-agent drugs or when combined with cisplatin. H3k4me2 was significantly increased by TSA/cisplatin while H3K9me3 was slightly decreased when exposed to TSA/cisplatin or TSA (Figure 3B). 
<smiles>CC(/C=C/C(=O)NO)=C\C(C)C(=O)c1ccc(N(C)C)cc1</smiles>

Trichostatin A (TSA)

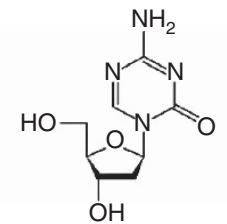

5-aza-2'-deoxycytidine (5-aza-CdR)
B

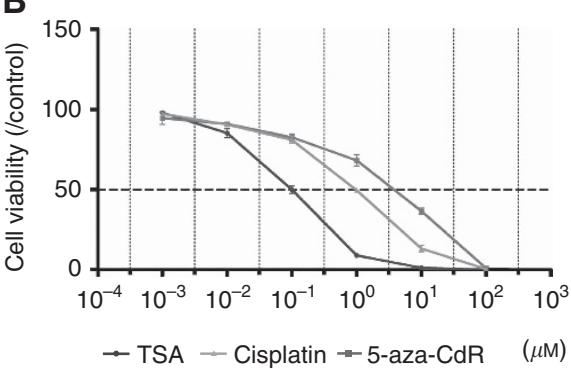

C

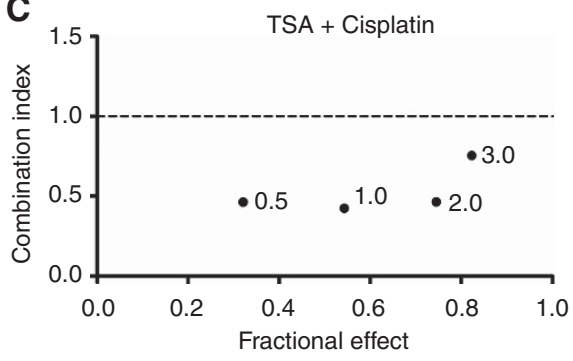

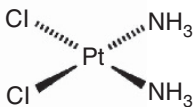

Cisplatin

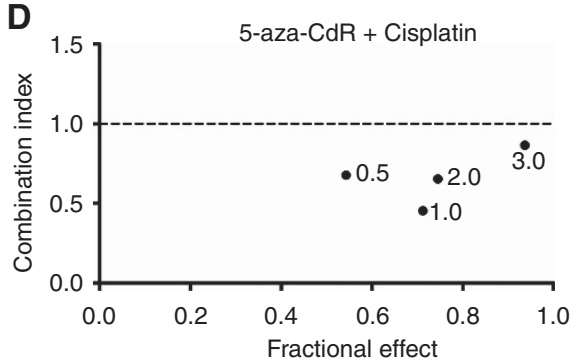

Figure 1. Effect of TSA, 5 -aza-CdR, and cisplatin alone or in combination on the cell viability of ovarian cancer cell lines. (A) The chemical structure of TSA, 5-aza-CdR, and cisplatin. (B) Dose-response curve of SKOV3 cells in the presence of increasing doses of drugs for $48 \mathrm{~h}$ in vitro. The experiment was performed in triplicate and the data were presented as mean \pm s.e.m. Synergism was investigated by exposing SKOV3 cells to a fixed ratio of (C) TSA and cisplatin $(0.3: 1)$ or (D) 5 -aza-CdR and cisplatin $(10: 1)$ and cell viability were detected by MTS assay. The numbers labelled indicate the fraction (0.5) and multiples $\left(1.0,2.0\right.$, and 3.0) of the $I C_{50}$ concentration in a fixed ratio $(T S A / c i s p l a t i n=0.3 / 1 ; 5$-aza-CdR/ cisplatin $=10 / 1)$. Combination index $<1$ indicates synergistic effect. All dosages had a $\mathrm{Cl}<1$.

Spheroids as ovarian cancer in vitro drug screening tool and the effect of drugs on spheroid formation and growth. Spheroids are found normally occurring in human ascites and were grown in vitro from three cancer cells lines HEY, SKOV3, and A2780 as well as the normal cell line IOSE. These spheroids grow on nonadherent plates and form compact spheres (Figure 4A). Epithelial to mesenchymal transition markers Twist, Slug, Snail, and $\mathrm{N}$-cadherin were all upregulated when cells were grown as spheroids while the mesenchymal to epithelial (MET) marker E-cadherin was downregulated in spheroid culture (Figure 4B). Spheroids may represent an important factor in the dissemination and colonisation of ovarian cancer to the omentum in ovarian cancer patients. SKOV3 cells were investigated to assess the effect of these drugs on spheroid formation and growth. Both high-dose TSA $(600 \mathrm{~nm})$ and 5-aza-CdR $(10 \mu \mathrm{M})$ showed similar inhibition of spheroid formation as were observed in the lower doses of TSA (300 $\mathrm{nm})$ and 5-aza-CdR $(5 \mu \mathrm{M})$ when combined with cisplatin (Supplementary Figure S2). Spheroid number was also suppressed by each drug alone in all three cancer cell lines. Trichostatin $\mathrm{A} /$ cisplatin had the same inhibitory effect on spheroid formation as did high-dose TSA (600 nM) in SKOV3 cells (Figure 4C) and A2780 cells (Supplementary Figure S2B). 5-aza-2'-deoxycytidine had less effect on spheroid formation than TSA in SKOV3 (Figure 4C) and A2780 (Supplementary Figure S2B) cells, but there was minimal response in the $\mathrm{HEY}$ cell line (Figure $4 \mathrm{C}$, Supplementary Figure S3). When drugs were added after spheroids had formed, spheroid growth and cell viability were markedly suppressed by TSA (300 nM and $600 \mathrm{~nm}$ ) and TSA/cisplatin (Supplementary Figure S4). IOSE spheroid assay showed that normal ovarian cells, which also have the ability to form spheroids, were minimally affected (Supplementary Figure S3C).

Sequential treatments of TSA, 5-aza-CdR, and cisplatin suppress the growth of tumour xenografts. Tumorgenicity of pretreated HEY cancer cells were assessed in SCID mice. The monotherapy with TSA, 5-aza-CdR, and cisplatin significantly inhibited tumour formation. The combination of TSA/cisplatin showed more tumour suppression than monotherapy and was more effective than the combination of 5 -aza-CdR/cisplatin in terms of tumour volume and weight (Supplementary Figure S5). We next investigated the effect of sequential in vivo treatments of TSA, 5 -aza-CdR, and cisplatin on HEY tumour xenograft (Figure 5A). Differences between tumour weight (Figure 5B) and volume (Figure 5C) suggested that cisplatin followed by TSA was the most effective approach to suppress tumour growth. 5-aza-2'-deoxycytidine followed by cisplatin was more effective in suppressing tumour growth than cisplatin followed by 5-aza-CdR. The expression of EMT markers Twist, Slug, and Snail were significantly suppressed by sequential treatment of TSA and cisplatin and vice versa (Figure 5D). Only cisplatin followed by TSA suppressed $\mathrm{N}$-cadherin, while 5-aza-CdR/cisplatin and cisplatin/5-aza-CdR actually increased $\mathrm{N}$-cadherin expression. All sequential treatments slightly enhanced the expression of E-cadherin (Figure 5D). Pluripotency markers Oct4 and Sox2 were markedly suppressed by all sequential treatments while Nanog was only significantly suppressed with 5 -aza-CdR followed by cisplatin (Figure 5D). Sequential treatment of low-dose cisplatin followed by TSA demonstrated the most tumour suppression without apparent animal toxicity (Figure 5E).

\section{DISCUSSION}

In this study, we have investigated the optimal combination of cisplatin and two epigenetic modifiers TSA and 5-aza-CdR in cell culture and xenografts. We found that TSA and 5-aza-CdR, in combination with low-dose cisplatin significantly inhibited spheroid formation and tumorigenicity via the suppression of EMT and pluripotency of ovarian cancer cells, both in vitro and in vivo, with the maximal effect with cisplatin followed by TSA in the animal model. In addition, in our in vitro work, we found that both epigenetic modifiers increased the sensitivity of the cancer cell lines to cisplatin, suggesting that they may have an important role in platinum-resistant ovarian cancer.

Abdominal dissemination of tumour cells is one of the main metastatic routes of ovarian cancer, so the ability to migrate, adhere, and self-renew are critical to ovarian cancer metastasis 

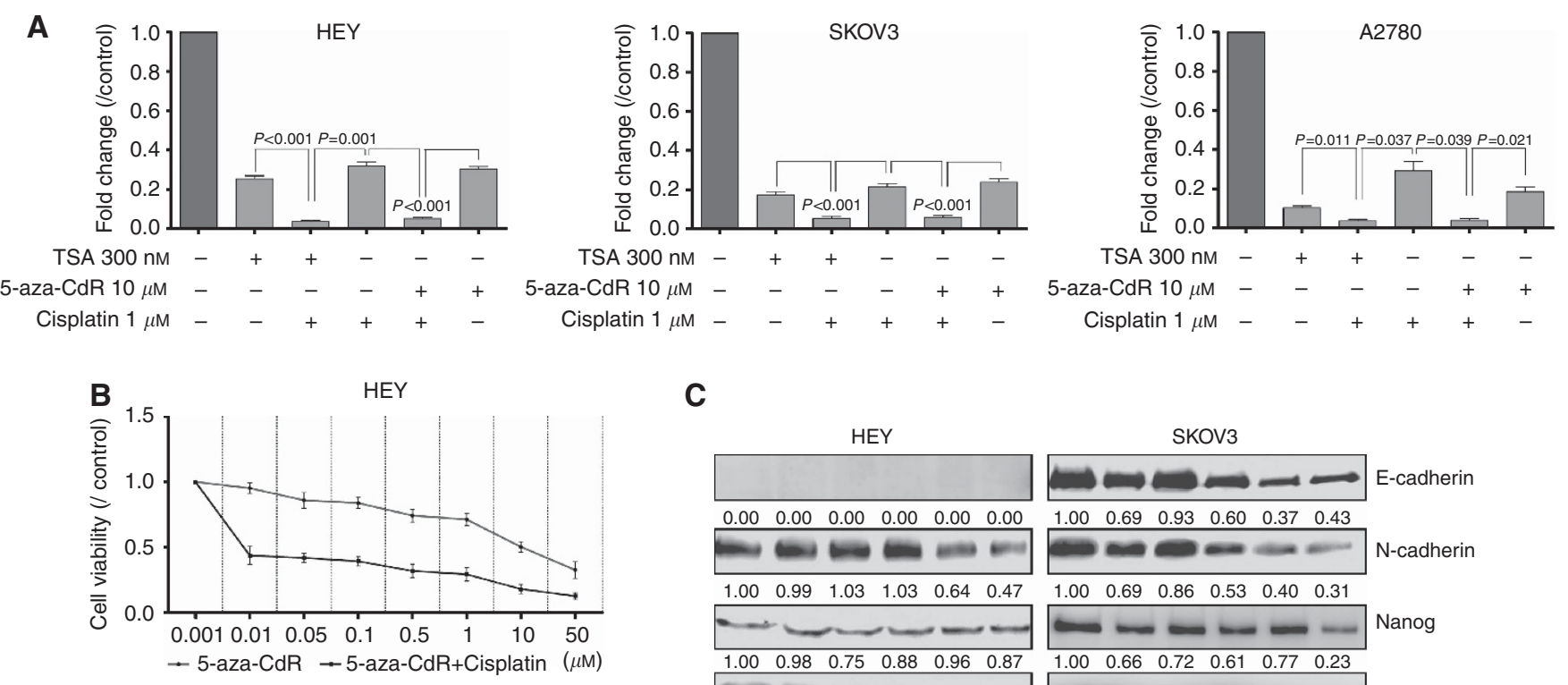

C
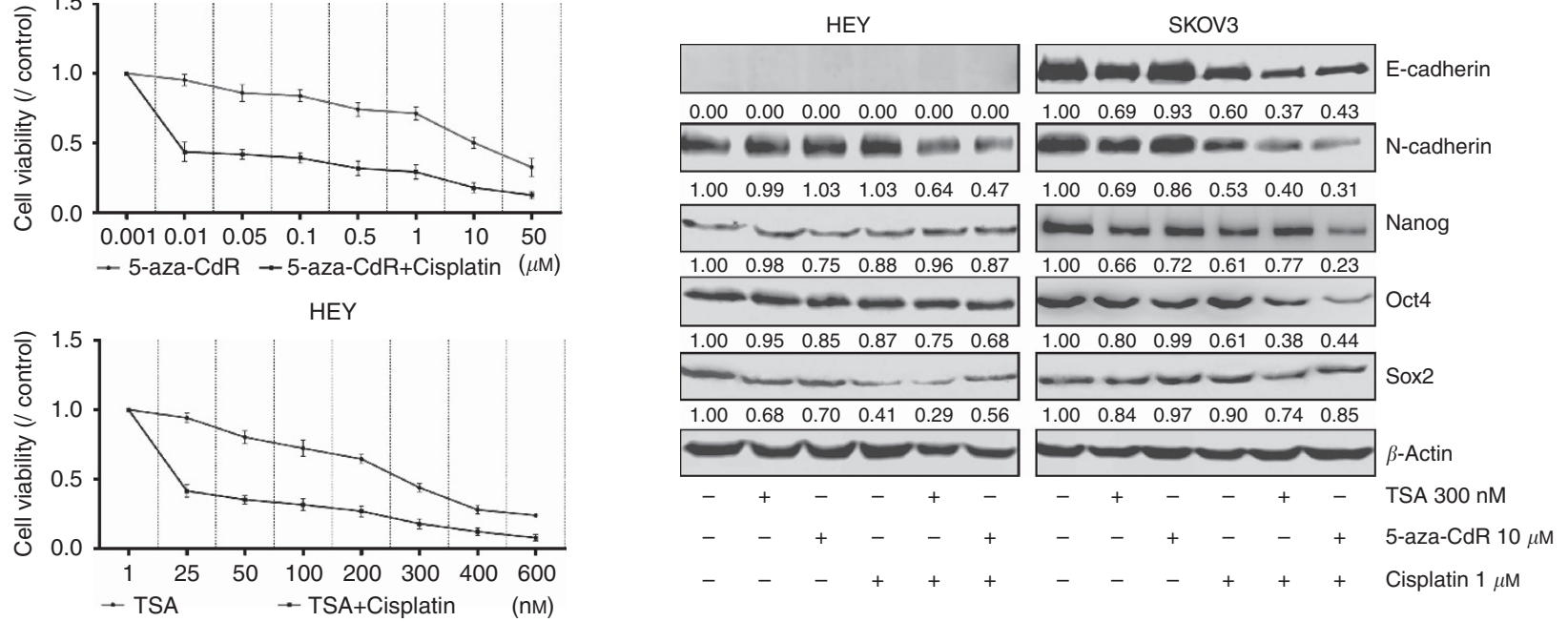

Figure 2. Effect of TSA, 5-aza-CdR, and cisplatin alone or in combination on cell migration, chemosensitivity, and the expression of important proteins associated with EMT and pluripotency. (A) Quantitative analysis of migration of ovarian cancer cells (HEY, SKOV3, and A2780) when treated with TSA, 5-aza-CdR, and cisplatin alone or in combination expression as fold change compared with the untreated cells, mean \pm s.e.m., $n=5$ (B) Chemotherapy sensitisation assay with HEY cells treated with a fixed dose of cisplatin (1 $\mu \mathrm{m})$ with increasing doses of TSA or 5 -aza-CdR measured by cell viability. Each point represents mean \pm s.e.m. (C) HEY and SKOV3 cells were exposed to the same drugs as the migration assay and the expression levels of proteins were detected by western blot, $\beta$-actin is a loading control. Quantitation was done using density of the blots and was a percentage compared with the control cells.

A

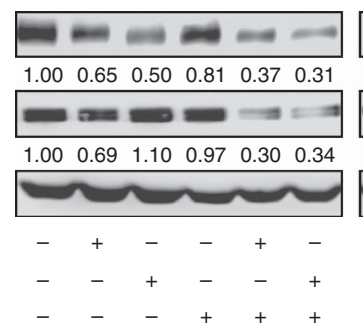

$\mathrm{A} 2780$

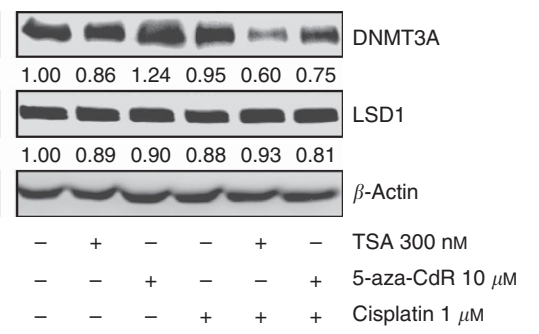

B

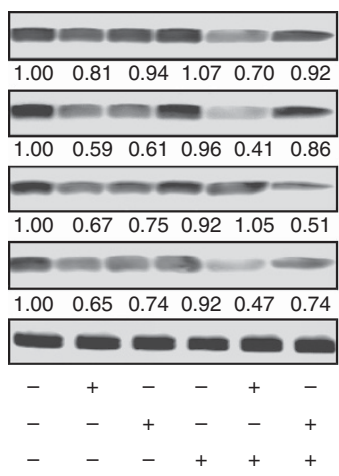

A2780

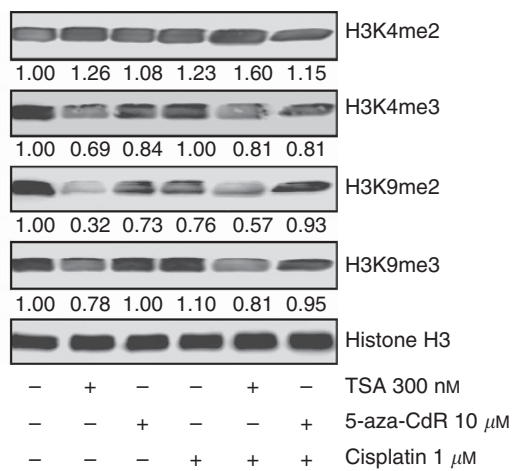

Figure 3. Epigenetic regulation of ovarian cancer cells in the presence of different drugs. (A) Effect of treatment on the expression of epigenetic regulation enzymes DNMT3A and LSD1 in SKOV3 and A2780 cells. Both cells lines were treated for $48 \mathrm{~h}$ in vitro and the expression of DNMT3A and LSD1 were detected by western blot. (B) Histone methylation markers H3K4me2/me3 and H3K9me2/me 3 were also measured in SKOV3 and A2780 cells. Histone $\mathrm{H} 3$ is the loading control.

(Onder et al, 2008; Sawada et al, 2008). Twist and Snail are transcription factors that regulate the expression E-cadherin and $\mathrm{N}$-cadherin. Ovarian cancer patients with high expression of Twist and Snail have poorer progression-free survival and overall survival, suggesting that cadherin expression impacts survival (Hosono et al, 2007; Blechschmidt et al, 2008). In our study,
$\mathrm{N}$-cadherin and transcription factors Twist, Slug, and Snail were suppressed and E-cadherin was induced by the sequential treatment of cisplatin followed by TSA in the xenografted animal model, showing that the combination of epigenetic modifiers and cisplatin could reverse the EMT in vivo and suppress tumour growth. Interestingly, in our cell line data, SKOV3, which is 
A
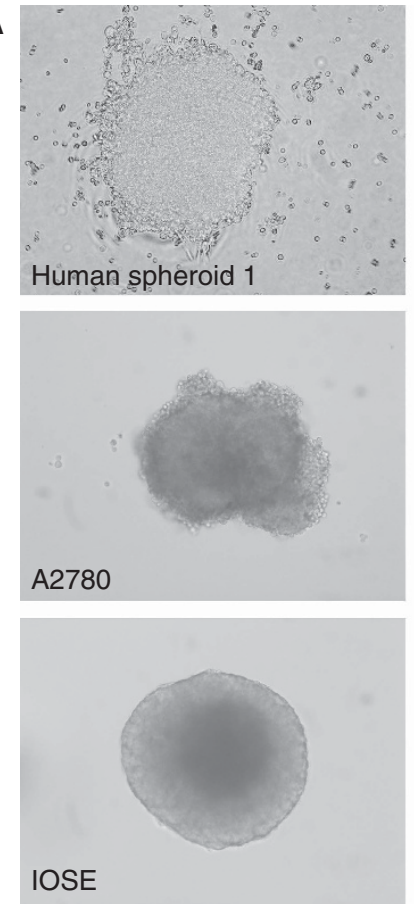
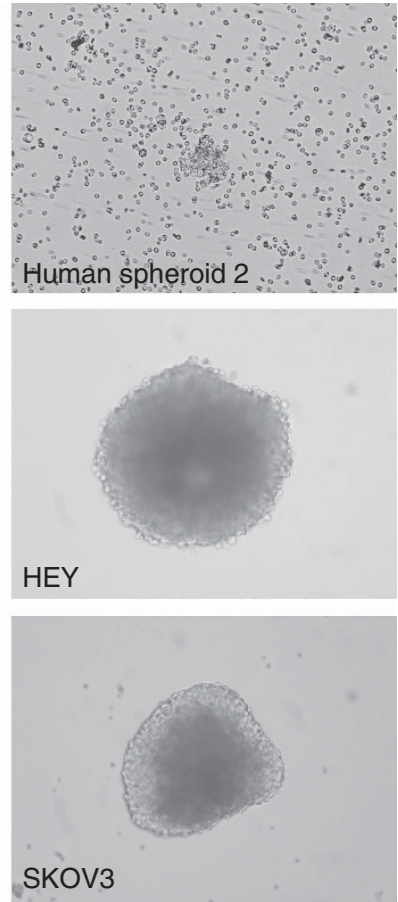

B

\section{SKOV3}
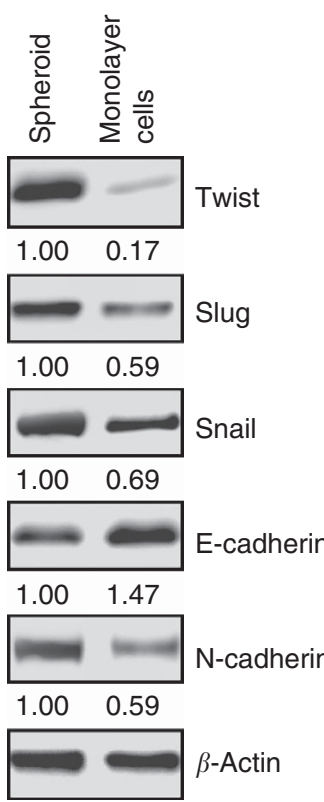

C

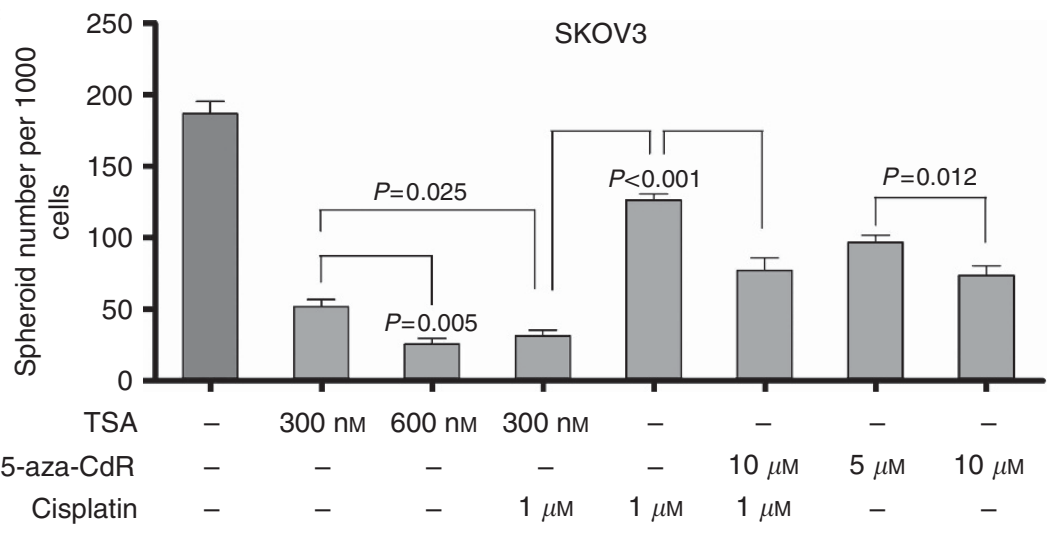

Figure 4. Inhibitory effect of TSA, 5-aza-CdR, and cisplatin alone or in combination on the formation and growth of spheroids. (A) Representative images of normally occurring spheroids in malignant ascites and spheroids grown in vitro from ovarian cancer cell lines HEY, SKOV3, A2780, and normal epithelium IOSE at $\times 100$. (B) The expression of EMT markers of spheroid and parental cells were assessed by western blot. (C) Effect of different concentration of TSA, 5-aza-CdR, and cisplatin alone or in combination on spheroid number in SKOV3 cells.

derived from ascites, showed downregulation of both E-cadherin and $\mathrm{N}$-cadherin with exposure to cisplatin and TSA, and cisplatin and 5-aza-CdR, suggesting that the combination may promote anticancer activity primarily via the suppression of E-cadherin. Clearly, the role of E-cadherin in ovarian cancer needs further investigation. The inhibitory effect on pluripotency markers suggests that inhibition of cell renewal is another important mechanism of the combination of epigenetic modifiers and the conventional chemotherapy agent cisplatin in ovarian cancer (Hu et al, 2010; Ma et al, 2010; Pan et al, 2010; Peng et al, 2010; Ye et al, 2011).

Ovarian cancer cells are found in the peritoneal cavity as multicellular spheroid aggregates and are resistant to radiation and chemotherapy, which allow them to survive cytotoxic treatment. These compact spheroids have an increase in intercellular integrins and fibronectin, which are thought to promote adhesion and invasion of ovarian cancer cells in distant sites (Burleson et al, 2004; Bapat et al, 2005; Burleson et al, 2006; L'Espérance et al, 2008; Zhang et al, 2008). Thus, prevention of spheroid formation and growth represents a novel strategy to improve the efficacy of current intraperitoneal chemotherapy by more effectively treating these resistant aggregates. We hypothesise that EMT has a pivotal role in spheroid formation and survival, and treatment regimens that reverse this process would be the key to the prevention of ovarian cancer metastasis and recurrence. We have also found an inhibitory effect of the combination of TSA and 5-aza-CdR on spheroid formation through suppression of EMT and ECM degradation (data not shown), supporting this hypothesis.

Epigenetic abnormalities have been detected in many cancers, including ovarian cancer, and are thought to be one of the most important mechanisms of tumorigenesis (Wei et al., 2006; Tsai, Baylin 2011). Reversing these changes has significant appeal as alternatives to or in addition to traditional cytotoxic therapy. Our findings show that the combination of epigenetic modifiers and conventional chemotherapeutic agents is more effective than each single drug alone (Shaker et al, 2003; La Rosée et al, 2004). DNMT3A and LSD1 are both overexpressed in many cancer cells and are related to a poor prognosis (Caslini et al, 2006). Suppression of these critical pathways in tumorigenesis holds great promise to more effectively treat these cancers. Combination 


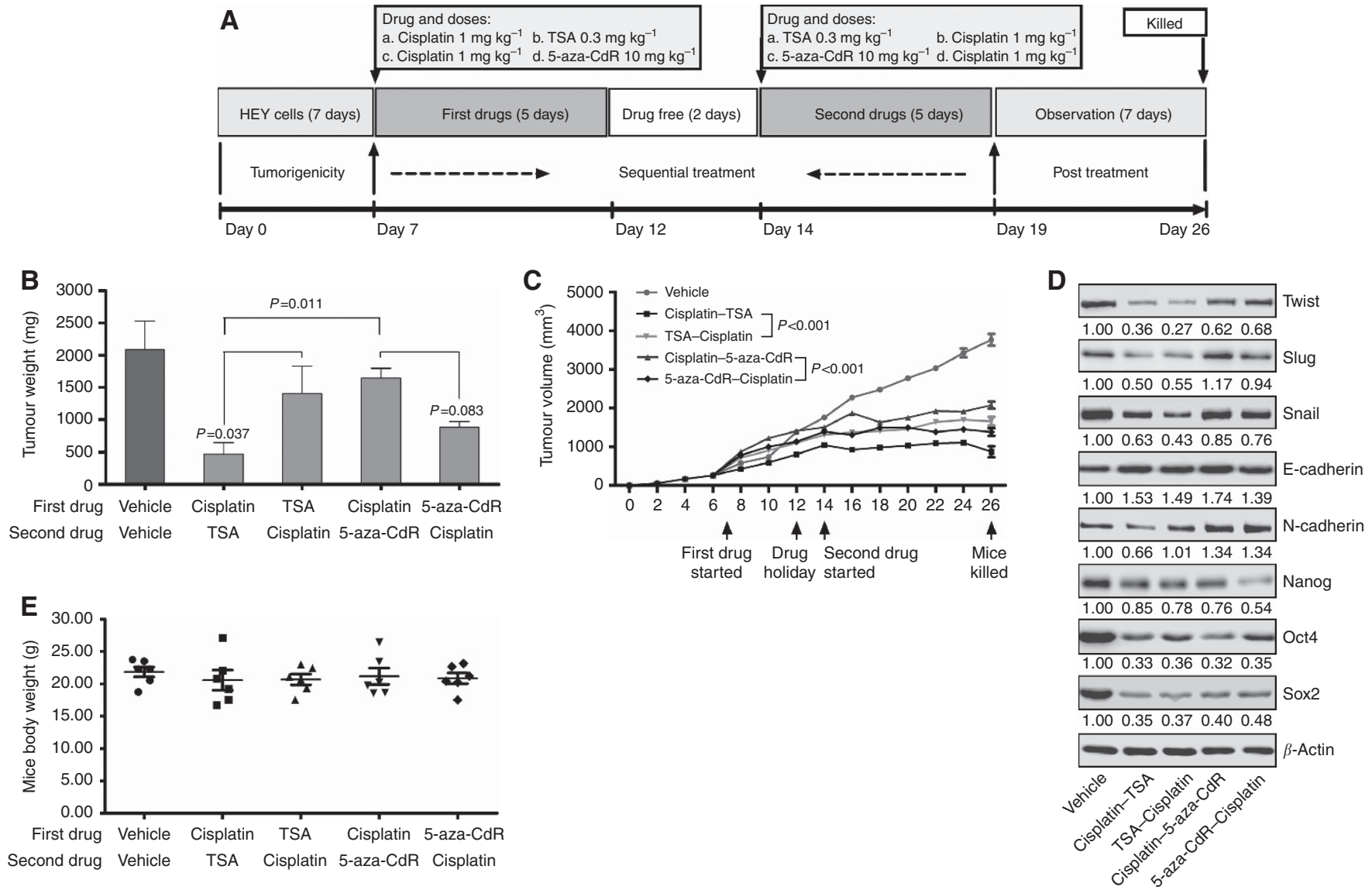

Figure 5. Effect of sequential drug administration in tumour xenograft mice model (A) The scheme of sequential treatment strategies. All drugs were administrated at the same time point on each treatment day. (B) Tumour weights were evaluated at day 26 , the results presented as mean \pm s.e.m., $P<0.05$ denotes statistical significance. (C) Tumour volume was measured and calculated for each treatment group. (D) The expression of EMT markers and pluripotency markers from tumour were detected by western blot. $\beta$-actin is a loading control. (E) The mice were weighed at day 26 and compared between treatment groups. $n=6$.

treatment achieved broad-spectrum histone modification as well as crosstalk between histone acetylation, DNA methylation, and histone methylation (Cedar et al, 2009). Our results suggest that there exist complicated interactions in ovarian cancer cells between these pathways; understanding the map of epigenetic abnormalities and interaction of different epigenetic regulators may be helpful in developing new chemotherapeutic targets for ovarian cancer.

The fact that the sequential treatment of TSA and cisplatin resulted in significant reduction of tumorigenesis suggests that cancer cells become more sensitive to lower doses of cytotoxic chemotherapy when treated with epigenetic modifiers. This approach could have a substantial impact on women with platinum-resistant disease where few effective treatment options exist. Limitations of this study include the short duration of treatment with these drugs and the use of sequential rather than concomitant treatment in our animal model. Further research to determine optimal doses, treatment time, and regimens need to be done before translating this approach to patient care. This study provides new and promising approaches for platinum-resistant ovarian cancer with far less toxicity than current regimens.

\section{ACKNOWLEDGEMENTS}

Grant support was provided by The Carole and Ray Neag Comprehensive Cancer Center and The University of Connecticut Health Center. We thank Dr Susan Murphy (Obstetrics and
Gynaecology, Duke University Medical Center, NC, USA) for sharing the A2780 cell line.

\section{CONFLICT OF INTEREST}

The authors declare no conflict of interest.

\section{REFERENCES}

Ahmed N, Thompson EW, Quinn MA (2007) Epithelial-mesenchymal interconversions in normal ovarian surface epithelium and ovarian carcinomas: an exception to the norm. J Cell Physiol 213: 581-588.

Asadollahi R, Hyde CA, Zhong XY (2010) Epigenetics of ovarian cancer: from the lab to the clinic. Gynecol Oncol 118: 81-87.

Baba T, Convery PA, Matsumura N, Whitaker RS, Kondoh E, Perry T, Huang Z, Bentley RC, Mori S, Fujii S, Marks JR, Berchuck A, Murphy SK (2009) Epigenetic regulation of CD133 and tumorigenicity of CD133 + ovarian cancer cells. Oncogene 28: 209-218.

Balch C, Fang F, Matei DE, Huang TH, Nephew KP (2009) Minireview: epigenetic changes in ovarian cancer. Endocrinology 150: 4003-4011.

Bapat SA, Mali AM, Koppikar CB, Kurrey NK (2005) Stem and progenitor-like cells contribute to the aggressive behavior of human epithelial ovarian cancer. Cancer Res 65: 3025-3029.

Bast Jr RC, Hennessy B, Mills GB (2009) The biology of ovarian cancer: new opportunities for translation. Nat Rev Cancer 9: 415-428.

Blechschmidt K, Sassen S, Schmalfeldt B, Schuster T, Höfler H Becker KF (2008) The E-cadherin repressor Snail is associated with 
lower overall survival of ovarian cancer patients. Br J Cancer 98: 489-95.

Burleson KM, Boente MP, Pambuccian SE, Skubitz AP (2006) Disaggregation and invasion of ovarian carcinoma ascites spheroids. $J$ Transl Med 24: 4-6.

Burleson KM, Hansen LK, Skubitz AP (2004) Ovarian carcinoma spheroids disaggregate on type I collagen and invade live human mesothelial cell monolayers. Clin Exp Metastasis 21: 685-697.

Caslini C, Capo-chichi CD, Roland IH, Nicolas E, Yeung AT, Xu XX (2006) Histone modifications silence the GATA transcription factor genes in ovarian cancer. Oncogene 25: 5446-5461.

Cedar H, Bergman Y (2009) Linking DNA methylation and histone modification: patterns and paradigms. Nat Rev Genet 10: 295-304.

Chou TC (2010) Drug combination studies and their synergy quantification using the Chou-Talalay method. Cancer Res 70: 440-446.

Fang F, Balch C, Schilder J, Breen T, Zhang S, Shen C, Li L, Kulesavage C, Snyder AJ, Nephew KP, Matei DE (2010) A phase 1 and pharmacodynamic study of decitabine in combination with carboplatin in patients with recurrent, platinumresistant, epithelial ovarian cancer. Cancer 116: 4043-4053.

Hosono S, Kajiyama H, Terauchi M, Shibata K, Ino K, Nawa A, Kikkawa F (2007) Expression of Twist increases the risk for recurrence and for poor survival in epithelial ovarian carcinoma patients. Br J Cancer 96: 314-320.

Hu L, McArthur C, Jaffe RB (2010) Ovarian cancer stem-like side-population cells are tumourigenic and chemoresistant. Br J Cancer 102: 1276-1283.

Kelly TK, De Carvalho DD, Jones PA (2010) Epigenetic modifications as therapeutic targets. Nat Biotechnol 28: 1069-1078.

Kristensen LS, Nielsen HM, Hansen LL (2009) Epigenetics and cancer treatment. Eur J Pharmacol 625: 131-142.

L'Espérance S, Bachvarova M, Tetu B, Mes-Masson AM, Bachvarov D (2008) Global gene expression analysis of early response to chemotherapy treatment in ovarian cancer spheroids. BMC Genomics 9: 99.

La Rosée P, Johnson K, Corbin AS, Stoffregen EP, Moseson EM, Willis S, Mauro MM, Melo JV, Deininger MW, Druker BJ (2004) In vitro efficacy of combined treatment depends on the underlying mechanism of resistance in imatinib-resistant Bcr-Abl-positive cell lines. Blood 103: 208-215.

Lengyel E (2010) Ovarian cancer development and metastasis. Am J Pathol 177: 1053-1064.

Ma L, Lai D, Liu T, Cheng W, Guo L (2010) Cancer stem-like cells can be isolated with drug selection in human ovarian cancer cell line SKOV3. Acta Biochim Biophys Sin 42: 593-602.

Onder TT, Gupta PB, Mani SA, Yang J, Lander ES, Weinberg RA (2008) Loss of E-cadherin promotes metastasis via multiple downstream transcriptional pathways. Cancer Res 68: 3645-3654.

Pan Y, Jiao J, Zhou C, Cheng Q, Hu Y, Chen H (2010) Nanog is highly expressed in ovarian serous cystadenocarcinoma and correlated with clinical stage and pathological grade. Pathobiology 77: 283-288.

Peng S, Maihle NJ, Huang Y (2010) Pluripotency factors Lin28 and Oct4 identify a sub-population of stem cell-like cells in ovarian cancer. Oncogene 29: 2153-2159.

Plimack ER, Stewart DJ, Issa JP (2007) Combining epigenetic and cytotoxic therapy in the treatment of solid tumors. J Clin Oncol 25: 4519-4521.
Sawada K, Mitra AK, Radjabi AR, Bhaskar V, Kistner EO, Tretiakova M, Jagadeeswaran S, Montag A, Becker A, Kenny HA, Peter ME,

Ramakrishnan V, Yamada SD, Lengyel E (2008) Loss of E-cadherin promotes ovarian cancer metastasis via alpha 5-integrin, which is a therapeutic target. Cancer Res 68: 2329-2339.

Shaker S, Bernstein M, Momparler LF, Momparler RL (2003) Preclinical evaluation of antineoplastic activity of inhibitors of DNA methylation (5-aza-2'-deoxycytidine) and histone deacetylation (trichostatinA, depsipeptide) in combination against myeloid leukemic cells. Leuk Res 27: 437-444.

Siegel R, Naishadham D, Jemal A (2012) Cancer statistics, 2012. CA Cancer J Clin 62: 10-29.

Sodek KL, Ringuette MJ, Brown TJ (2009) Compact spheroid formation by ovarian cancer cells is associated with contractile behavior and an invasive phenotype. Int J Cancer 124: 2060-2070.

Su HY, Lai HC, Lin YW, Chou YC, Liu CY, Yu MH (2009) An epigenetic marker panel for screening and prognostic prediction of ovarian cancer. Int J Cancer 124: 387-393.

Teschendorff AE, Menon U, Gentry-Maharaj A, Ramus SJ, Gayther SA, Apostolidou S, Jones A, Lechner M, Beck S, Jacobs IJ, Widschwendter M (2009) An epigenetic signature in peripheral blood predicts active ovarian cancer. PLoS One 4: e8274.

Tsai HC, Baylin SB (2011) Cancer epigenetics: linking basic biology to clinical medicine. Cell Res 21: 502-517.

Vaughan S, Coward JI, Bast Jr RC, Berchuck A, Berek JS, Brenton JD, Coukos G, Crum CC, Drapkin R, Etemadmoghadam D, Friedlander M, Gabra H, Kaye SB, Lord CJ, Lengyel E, Levine DA, McNeish IA, Menon U, Mills GB, Nephew KP, Oza AM, Sood AK, Stronach EA, Walczak H, Bowtell DD, Balkwill FR (2011) Rethinking ovarian cancer: recommendations for improving outcomes. Nat Rev Cancer 11: 719-725.

Wei SH, Balch C, Paik HH, Kim YS, Baldwin RL, Liyanarachchi S, Li L, Wang Z, Wan JC, Davuluri RV, Karlan BY, Gifford G, Brown R, Kim S, Huang TH, Nephew KP (2006) Prognostic DNA methylation biomarkers in ovarian cancer. Clin Cancer Res 12: 2788-2794.

Yap TA, Carden CP, Kaye SB (2009) Beyond chemotherapy: targeted therapies in ovarian cancer. Nat Rev Cancer 9: 167-181.

Ye F, Li Y, Hu Y, Zhou C, Hu Y, Chen H (2011) Expression of Sox2 in human ovarian epithelial carcinoma. J Cancer Res Clin Oncol 137: 131-137.

Yoo CB, Jones PA (2006) Epigenetic therapy of cancer: past, present and future. Nat Rev Drug Discov 5: 37-50.

Zhang S, Balch C, Chan MW, Lai HC, Matei D, Schilder JM, Yan PS, Huang TH, Nephew KP (2008) Identification and characterization of ovarian cancer-initiating cells from primary human tumours. Cancer Res $\mathbf{6 8}$ : 4311-4320.

This work is published under the standard license to publish agreement. After 12 months the work will become freely available and the license terms will switch to a Creative Commons AttributionNonCommercial-Share Alike 3.0 Unported License.

Supplementary Information accompanies this paper on British Journal of Cancer website (http://www.nature.com/bjc) 